\title{
Pulse pressure trajectories in relation to cardiovascular mortality and dietary protein intake: the Zutphen Study
}

\author{
E. Mertens, S.M.A.J. Tielemans, S.S. Soedamah-Muthu and J.M. Geleijnse \\ Chair Group of Nutrition and Epidemiology, Division of Human Nutrition, Wageningen University, PO BOX 8129 , \\ 6700 EV Wageningen, the Netherlands
}

Findings from epidemiological studies suggested that higher dietary protein intake might have a beneficial influence on blood pressure (BP), and thereby lowering cardiovascular risk ${ }^{(1)}$. Also, pulse pressure (PP) is considered as an important physiological component of cardiovascular risk ${ }^{(2)}$, especially long-term patterns of BP appeared to be strongly associated with cardiovascular mortality ${ }^{(3)}$. The present study, therefore, aimed to characterise PP trajectories based on annual BP measurements over a 10 year period, and to investigate the association between PP trajectories and cardiovascular mortality using data from the Zutphen Study, a prospective cohort Study. Furthermore, the relationship between long-term protein intake and long-term patterns of PP will be examined.

Included in the present analysis were 632 middle-aged men, of whom BP was annually measured from 1960 until 1970, and who were followed until death. PP was calculated as the difference between systolic and diastolic BP. PP trajectories based on annual BP measurements were identified by using latent class growth analysis, and were examined in relation to cardiovascular mortality (Cox proportional hazard analysis) with adjustments for age, serum cholesterol level, smoking and diabetes. Habitual dietary intake was measured three times at a five year interval by a cross-check dietary history. For the present study, dietary protein intake was considered as total protein, protein origin (plant and animal), and protein from main food sources (grain, dairy and meat). To investigate the association between repeatedly measured dietary protein intake and PP trajectories linear mixed models, especially a random coefficients model with random intercept and slope with time, was applied, in order to obtain adjusted absolute protein values. Adjustments were made for energy intake, lifestyle factors and nutrient intakes.

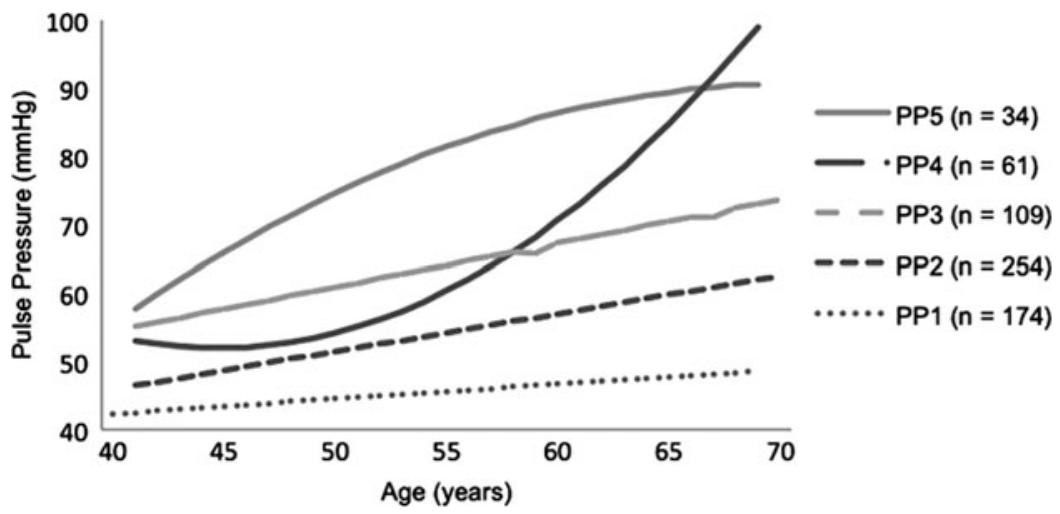

Figure 1. Five 10-year trajectories of the pulse pressure.

Five 10-year PP trajectories were identified, representing the increase of PP from middle-age through old age by five different patterns over time (Figure 1). The mean increase in PP from 50 to 60 years varied from 2 (lower-level PP trajectory, PP1) to $15 \mathrm{mmHg}$ (higher-level PP trajectories, PP4 and PP5). The higher-level PP trajectories were associated with a significant $2 \cdot 3-3 \cdot 4$ times higher risk for cardiovascular mortality compared with the lowest-level trajectory after adjustment. In contrast, total dietary protein was not related to PP trajectories, with all adjusted protein intake being close to $88 \mathrm{~g} / \mathrm{d}$. Additionally, no clear associations were observed for plant and animal protein, and protein from grain, dairy and meat.

In conclusion, five 10-year PP trajectories were identified in the Zutphen Study among 632 Dutch middle-aged men, which were strong predictors of cardiovascular mortality. Intake of total protein was not associated with PP trajectories, and no association was found for origin and main sources of protein intake. Future research should examine whether these findings can be generalised to women, because there may be gender-specific differences in arterial ageing, and thus PP.

1. Rebholz C.M., et al. (2012) Am. J. Epidemiol. 176, S27-S43.

2. Lee H.-Y. \& B.-H. Oh (2010) Circulation 74, 2257.

3. Allen N.B., et al. (2014) Jama 311, 490-497. 